\title{
An Eye Tracking Study of Viewing Behaviour and Preferences of Arabic, English and Chinese Users
}

\author{
Mohammad Alsaffar, Lyn Pemberton, Karina Rodriguez Echavarria \\ University of Brighton, UK \\ Email: M.Alsaffar@brighton.ac.uk \\ Email:Lyn.Pemberton@brighton.ac.uk \\ Email: K.Rodriguez@brighton.ac.uk
}

\begin{abstract}
The aim of this research is to investigate possible variations in visual behaviour and eye movements on webpages among users from three different cultures (Arab, English and Chinese). The paper reports an experiment using eye tracking technique and questionnaire to understand how users from different countries fixate on webpages with different layouts and which type of layout they preferred. Thirty users from each group were exposed to three types of webpage (image-based, text-based and a webpage with unfamiliar design). The results indicated similarities and differences in fixations between the three cultural groups. Dissimilar scanning patterns were exhibited from each group. This study also revealed differences in preferences across Arab, English and Chinese users. This study contributes to the field of Human Computer Interaction by providing new insights into the similarities and differences between the users from the Middle East, the West and the Far East from the perspective of visual behaviour and preferences.
\end{abstract}

Visual behaviour, Web page, Eye tracking, Eye movement, Arab, English, Chinese, preferences.

\section{INTRODUCTION}

The exponential growth in the proliferation of information and communication technologies, particularly after the emergence of the Internet, demands greater consideration to aspects of websites' interface keeping international audiences in mind. The natural dissimilarities found in the culturally influenced traits of Internet users such as attitudes, attention and cognition makes presenting understandable and appropriate World Wide Web interfaces even more challenging. Cultural differences, particularly language differences, among global Internet users may have a significant impact on how users perceive a website. Consequently, the universal applicability of wellknown usability considerations on interface design cannot necessarily be trusted and can be questionable.

Earlier studies (Ahmed et al. 2009; Cyr et al. 2010; Cyr 2013) suggested that some users may find a web element clear, meaningful and understandable while, on the contrary, other users may find it ambiguous, arbitrary and meaningless. In addition, users' behaviour and attitude when browsing websites can be influenced by website design considerations (Lee \& Kozar 2012). Many studies examined the viewing behaviour on websites with different configurations by taking the approaches of website complexity criteria such as (Deng \& Poole 2010; Reinecke et al. 2013; Wang et al. 2014). Web design components such as headings, colours, pictures, and layout can be used effectively to attract users' attention (Reinecke et al. 2014). Attention can be defined as the allocation of cognitive resources to a visual stimulus by a person (Hoyer \& MacInnis 1997).

Websites developed throughout the world are used by local and global users. Users' reactions to the websites can differ in different ways based on their cultural characteristics. For example, certain elements within a website may attract more attention than others: some users might find a website overwhelming and consequently leave quickly (Faiola \& Matei 2005). However, studies concerning the presentation of websites and their impact on people's language and cognitive differences are scarce. Additionally, no controlled studies have been carried out previously to understand the differences in eye movement based on different language orientations and visual behaviour of culturally diversified users when exposed to different websites. Further, the visual behaviour of the users with the ability to read in 
multi-directions, e.g. (right to left and left to right) also needs to be investigated.

This study explores different users' attention to different website presentations, for instance, identifying whether the number of images on a website influences users' eye movement and satisfaction across cultural groups. This research aims to address the following questions:

- Do Arabic, English and Chinese users have different eye movement patterns when browsing different types of webpage?

- Do Arabic, English and Chinese users have different preferences for website interfaces?

To address these research questions, we used an eye tracking technology and a questionnaire to explore how different users with different cultural backgrounds and reading directions browse three types of interfaces. Human behaviour such as reading, scanning and processing of visual stimuli has been explored by researchers using eyetracking technology in the past few decades (Sharma \& Dubey 2014). The purpose of this study is not to examine the usability of websites but to utilise them as stimuli to understand the viewing behaviour of those users when interacting with similar website designs. Better understanding of global users' visual behaviour can result in increased performance and user satisfaction, which consequently benefit web design and usability considerations. The rest of the paper is organised as follows. Section 2 presents the background and some relevant studies related to website design. Section 3 describes the methodology employed in this sudy. Section 4 presents the results obtained from the experiment and section 5 presents a discussion and our conclusions from this study.

\section{BACKGROUND AND RELATED WORK}

Since the industrialisation of information and communication technologies begin in North America, users from that region were the main consideration of designers in most early websites interfaces (Alex 2014). Statistically, over $52 \%$ of all the websites exists in the world are in the English language (Web Technology Surveys 2018). The use of the Internet by non-native-English-speakers has increased significantly (Brandtzæg et al. 2011). However, websites that are designed in one part of the world may not meet the needs of users from other parts of the world. Organisations targeting customers internationally should consider various kinds of diversities among the users of their websites. According to (Hernandez et al. 2017), English, being the universal language for business, has been used in tandem with the local languages of the home countries by international marketers in global settings. Considerations such as cognitive styles, preferences and visual behaviour of potential users of a website need to be taken into account when designing interfaces to increase global acceptance (Hsieh 2008). More recently, most of the companies with an online presence aim to attract users' attention from all over the globe. Therefore, their interface designers attempt to improve user experience, performance and content relevance (Marcos \& Gavin 2015). It is equally important for companies to design website interfaces responsive to the global users' cultural diversifications, dissimilar visual behaviour and preferences.

\subsection{Website Design and Its influence}

A wealth of research has been carried out in the field of usability of interface, design process and the influence of culture on interface design (Badre 2001; Vatrapu \& Pérez-Quiñones 2006; Li et al. 2007). On the other hand, studies to investigate the area of globally usable interfaces, especially user satisfaction from different regions and their preferences of website presentation remain scarce. Aesthetic research has progressed significantly in the $\mathrm{HCl}$ community since a seminal paper "What is beautiful is usable" (Tractinsky et al. 2000) was published. Idiosyncratic characteristics of interaction have attracted a lot of attention since the contentious claim that pragmatic qualities of user interfaces are influenced by aesthetic design. (Tuch et al. 2012) suggested that the overall perception of websites' users has proven to be significantly influenced by the construct of visual appeal. (Lindgaard et al. 2011) concluded that stable visual appeal judgements can be established by the users in as short a time as $50 \mathrm{msec}$.

Since it will be impossible to recognise other elements of the website, such as content, these judgments are solely based on visual design. The constructs of perceived credibility and usability could also be established through relatively stable judgments in a similar exposure time (Lindgaard et al. 2011). The impression of beauty was examined to be the best predictor of overall judgment when a group of website users were studied for their first impression of a website (Schenkman \& Jonsson 2000) while the first impression itself can be considered as a predictor to determine whether a user will stay on a website or not. Furthermore, the balance of web page elements including colours plays an important role in influencing users' perception and preferences by providing users the sense of symmetry (Brady \& Phillips 2003). According to (Cyr 2008) users' trust, loyalty and satisfaction are correlated with the good visual design of a website. It is important to create a positive first impression as the negative impression can generally be very difficult to change.

Users' behaviour and attitude when browsing websites can be influenced by website design 
considerations (Lee \& Kozar 2012). In the recent years, researchers have noticed and highlighted the impact of website design, such as complexity, on users' behaviour and attitude towards website (Geissler et al. 2006; Deng \& Poole 2010; Tuch et al. 2009). While some researchers are of the opinion that simplicity within websites is key to achieve effectiveness (Venkatesh et al. 2003; Tuch et al. 2009), others have concluded that richness of information presentation within the complex design websites increases user satisfaction (Palmer \& W. 2002) so the contents of the websites remain the determining factor for users' overall experience of a website and also a predictor to stay on the website.

Investigating the effect of the structure of website presentation on website preferences and attention can be very helpful and useful to designers and marketing sectors to understand how the international audiences interact with such websites which in turns focus on a visual design issue that can be applied to attract users. The existence of diversified preferences across different cultures is well-known and this phenomenon influences users' perception of trust and satisfaction with a website (Head \& Cyr 2013). However, it remains unclear how the eye movement and preferences of users from different cultural backgrounds differ when browsing and viewing English websites with different configurations.

\subsection{The Influence of Website Aesthetics}

The aesthetics of user interface design have become an area of interest for user experience research carried out within the field of humancomputer interaction $(\mathrm{HCl})$. According to (Hartmann et al. 2007), overall user satisfaction has been influenced by aesthetic aspects over and above conventional usability factors. One factor of the aesthetic mix is colour. The feelings and reactions of website users can be impacted by the choice of colours. (Lindgaard et al. 2011), found that websites home pages having bright backgrounds rated higher than those of having dark backgrounds. Although the relationship between colours and individual's behaviours is not yet supported by a theoretical rationale, it has also been argued by (Bonnardel et al. 2011; Cyr et al. 2010; Palmer \& Schloss 2010) that previous studies have presented empirical evidence in favour of colours being influencer of individuals' behaviours such as attention, feelings, judgments and decisions of perceived usability. Different cultures have specific socially accepted and preferred colours. For example, in a study conducted by (Resort et al. 2009) on many Arabic University websites, colour schemes consists of blue and green as main colours found to be dominating. These two colours are frequent themes of Arabic visual communication along with the white background. In a similar study, (Palmer \& Schloss 2010) suggested that colours of higher saturation were found to be preferred over low saturation for Western adults for context-free patches of colour. Despite the differences across cultures, colour selections for website design were important to determine users' trust and satisfaction (Cyr et al. 2010). Cyr's study was based upon Canadian, German and Japanese cultures and analysed the relationship between cultures and consideration of colours for websites. Yellow was found to be the colour commonly rejected by the participants while the preferred colours varied across cultures, for example, blue for Germans and grey for Canadians.

The results of such studies carry great significance and relevance for marketers and website interface designers. On the other hand, (Reinecke \& Bernstein 2013) argue that visual complexity has greater significance compared to colours in the context of aesthetic perception of websites. They concluded that the selection of colours has a minor contribution in forming users' first impression of appeal, but the overall visual complexity mix has an overall impact on users' perception of a website. Findings from other studies also revealed that colours have a relatively low impact on visual aesthetics of a website compared to structural factors (Seckler et al. 2015). The number of studies into how the structure of website presentation influences users' attention and satisfaction is scarce. There is clearly still much to explore about the relationship between website design preferences and eye movement of users from different cultural backgrounds.

\section{METHODOLOGY}

The primary step of data collection was using eyetracking to record eye movements of subjects. It has been suggested that in comparative experiments, preferences should be collected in addition to eye-tracking (Cairns \& Cox 2008; Purchase 2012). Objective data related to the visual behaviour of participants was gathered using the eye-tracking system. Subjective data related to participants' preferences were collected via questionnaire. The overall visual stimulus was divided into different areas of interests (AOI's) such as top banner, bottom banner etc. for the purpose of statistical analysis. The eye-fixation is generally considered by researchers as the most relevant indicator to evaluate and process visual information acquisition. Other eye movement metrics, consistent with the other studies (Jacob \& Karn 2003; Pan et al. 2004), have also been used in this study. These include total fixation duration, time to first fixation and heatmaps.

Total fixation duration is the total duration of participants' fixation on a specific visual stimulus, while the average fixation duration indicates cognitive processing. Time to first fixation indicates 
the time taken from the user until notice a certain element on a stimulus. A heatmap is an objective measure based on the fixation duration of participants' attention of a webpage where red spots reflect most time fixated while the green and yellow show the areas of shorter fixation. Heatmaps are more useful when they are generated by the aggregated data collected from a number of participants to provide combined results.

\subsection{Apparatus}

The experiment was conducted using a Tobii Pro X2 eye tracking compact device screen-based which was connected to a 21 -inch monitor. The three webpages were displayed in Internet Explorer 9. The display resolution was set to $1280 \times 1024$. The Tobii Studio software was used to reduce and manipulate the raw data for further analysis.

\subsection{Preference data}

It has previously been suggested that for the purpose of eliciting more detailed answers from participants of different backgrounds, a "why" subheading should be added to the questionnaire (Jacko 2012; Purchase 2012; Lazar et al. 2017). After the trials of experimental part of the study, participants were asked to reflect on their preferences amongst the experimental conditions they were exposed to. This preference data was collected in addition to complement quantitative data gathered in the experiment.

\subsection{Participants}

This study comprised 90 participants divided in three groups of 30 members each. These groups were Arabs, English and Chinese. All of the participants were postgraduate or undergraduate students of the University of Brighton. This ensured that the participant had a minimum level of English proficiency required for this study. The mean ages of Arabic, English and Chinese participants were 32,31 and 30 respectively. There was no significant difference between the mean age of groups, as in a previous study (Rayner et al. 2006), differences between younger and older participants were discovered, with younger participants spending less time in reading compared to older participants. All the participants had previous experience of visiting various websites in English and were technologically proficient to take part in this study. All participants were rewarded with a $£ 6$ voucher for their participation.

\subsection{Study Design}

The purpose of this study was to investigate the differences in users' attention and eye movements by exposing different websites with different presentations. The aim was not to evaluate the presentation itself rather to elicit the potential differences in Arabic, English and Chinese users' attention and preferences when they viewed different types of website interfaces. Therefore, in order to generalise our findings, we chose three types of website presentations. It should be noted that we used only the homepages of these websites as stimuli in our experiment and from here on we will use word webpage instead of website, as the webpage means one page and the website is the collection of pages. The viewing behaviour was examined on these webpages only and not on the whole website. Webpage 1 was rich in images with less text, webpage 2 was rich with text only and webpage 3 was a mixture of text and images and also with unfamiliar structure of the presentation. For example, the logo and navigation bar of the webpage 3 were on the right hand side to obtain deep understanding about the diversity in users' eye movement and satisfaction.

Although randomisation took place during the experiment, each webpage was given a number to differentiate from each other. The three webpages were carefully selected after a two-step procedure. The first step was choosing three website presentations under each condition, based on our perceptions, so there were nine websites in total. The second step involved survey processing. Twenty students from different disciplines were exposed to the chosen webpages and two questions were asked after each website. The first question was about the familiarity with this webpage and the second question was about their feeling of the suitability of this website for its condition on 5 Likert scale $(5=$ very suitable/familiar and $1=$ not very suitable/familiar).

Once the data were gathered, the data were analysed statistically and the webpage that has less familiarity and high suitability was selected. This study was not to investigate how certain elements on the websites interfaces affect the visual behaviour of the users or examine the usability of it, but rather to explore how these users in reality viewing these webpages that contain a variety of design elements. Webpage 1 (see Figure 1 (a)) is the New Scientist website homepage from the website of the International science magazine and it includes many images all over the page. Webpage 2 (see Figure 1 (b)) is the Australia Government website, containing text only with no images. Webpage 3 (see Figure 1 (c)) is the Discover Southampton website consisting of text and images. It was important to make sure that none of the selected website was previously seen and known by the participants from the three groups. 


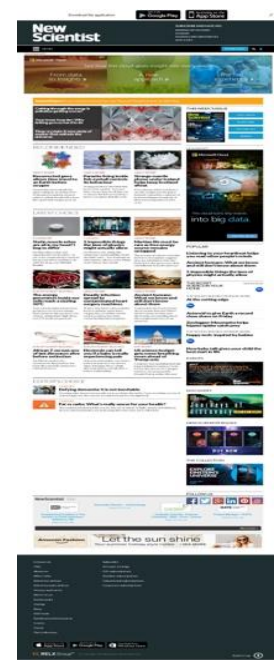

(a) Webpage1

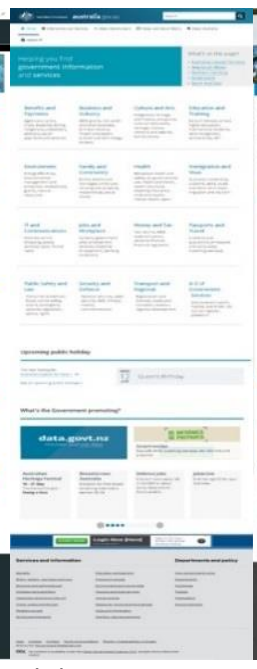

(b) Webpage2

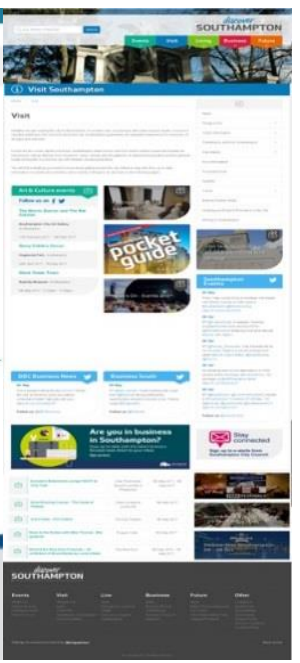

(c) Webpage3
Figure 1: The chosen webpages

According to (Cowen et al. 2002), using real webpages in the experiment is more acceptable and delivers more reliable findings than manipulating the webpage or creating a mock webpage for the purposes of the experiment. We selected real websites in order to provoke the natural attention and preferences from the three groups. In addition, in the experiment, the scrolling was allowed trying to make the users feel as in a real environment. Each stimulus from the tested websites has a different number of AOls based on its visual elements. There is no perfect sets of AOls for a stimulus and defining AOls always based on the researcher's intuition (Bojko 2013). As illustrated in Figure 2 ( $a, b$ and $c$ ) Webpage1 was divided into 22 AOls, Webpage2 was divided into $17 \mathrm{AOls}$ and Webpage3 has 19 AOls. Each $\mathrm{AOI}$ has a name that related to its position on the page. For example in Webpage3 there are three navigation bars in the website interface. Therefore, a position name was given to each one like Top Navigation, Right Navigation and Left Navigation to differentiate between them.

The participants were required to perform one task solely which was browsing freely the three types of websites interfaces with no certain goal or actions. The objective was to see how certain people actually see the webpage without searching for items in order to reveal their natural viewing behaviours and patterns. (A more task-oriented approach is reported in (Alsaffar 2018)).

\subsection{Procedure and Data Analysis}

Different exercise tasks were given to participants to get familiar with the eye-tracking device. The browsing task was only the task required from participants. Participants were required to browse the webpage freely for thirty seconds and in order to motivate them and to trigger their attention, a statement that includes a question was shown on the screen before seeing the stimuli. The statement

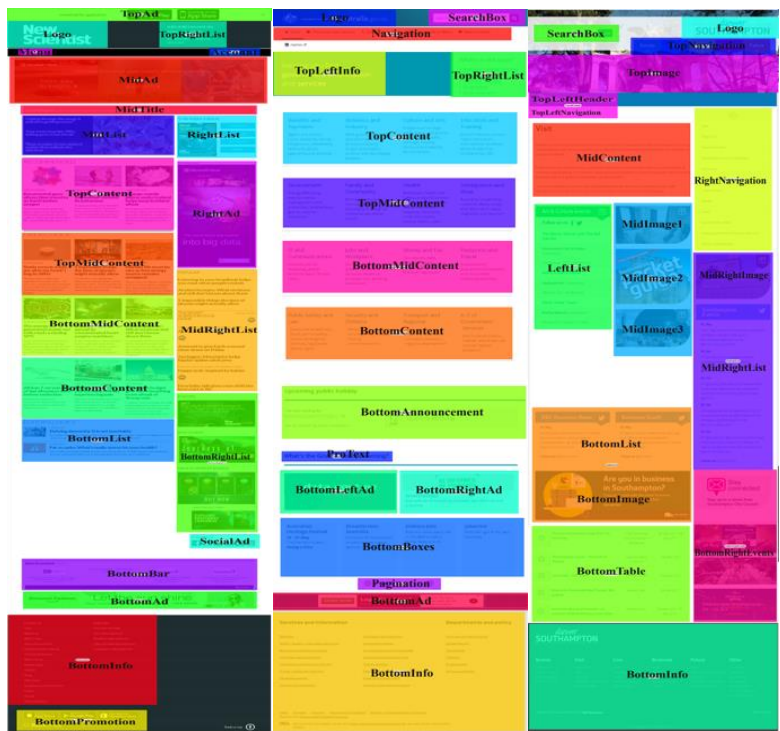

(a) Webpage1

(b) Webpage2

(c) Webpage3

Figure 2: The defined AOls for each webpage

was "You are going to see three different websites for about 30 seconds for each one. After viewing the three websites, you will be asked about the visual presentation (good or bad) and if you can express your opinions about the website presentation". The eye-tracking device was set to 30 seconds for each website to browse and the first 15 seconds of the eye movement data were collected and analysed only. This time frame was suggested by previous studies (Josephson \& Holmes 2002; Pan et al. 2004; Tzanidou 2006; Dong \& Lee 2008), which asserted that 15 seconds is sufficient to examine a webpage. Finally, participants were encouraged to answer the preferences question by choosing the most preferred type of website presentations and justifying their judgments.

It should be noted that before answering this question we explained for each Arabic, English and Chinese participant that choosing a preferred website interface depends on your vision and opinion in general and not necessarily what you have seen during the experiment in order for the results to be as general as possible. The collected data in this study came from two sources: eye tracking system and questionnaire. These data were analysed descriptively and inferentially and yielded in forms of graphs and tables to explore the phenomena. Quantitative statistical test such as Chi-Square was conducted on the preferences data beside the descriptive analysis. However, heatmaps add a qualitative flavour to the analysis by exemplifying the users' viewing patterns across the three webpages. The preferences data were analysed on two levels. The first level includes analysing participants' choices quantitatively and the second level includes analysing qualitatively their justifications of such a choice. Tobii Studio Software it combines with the Tobii Pro eye tracker device and it records the gaze data and provides 
statistical reports and presents visualised data such as heatmaps. In this study, each webpage was analysed and interpreted separately to provide a clear discussion for each one. The results are presented in two sections. The first section concerns the analysis results of gaze data that obtained from the eye tracking system. The second section focuses on presenting the preference data.

\section{EXPERIMENTAL RESULTS}

In the following subsections, four types of analysed data are presented: descriptive analysis of the total fixation duration, time to first fixation and the participants' fixation duration distributions using heatmaps for each group in each webpage. We then present the significant differences in the participants' preferences data.

\subsection{Fixations Analysis and Heatmaps in Webpage 1}

The webpage was divided into $22 \mathrm{AOls}$ as shown in Figure 2(a). Two metrics were analysed descriptively in this section; total fixation duration which calculates the total time of all fixations in each $\mathrm{AOI}$ and time to first fixation which indicates the time taken to notice each part of the webpage and in which order. One metric was analysed qualitatively which is heatmap which represents the aggregated fixation duration for each group. Table 1 presents the percentage of participants viewing each $\mathrm{AOI}$, total of fixation duration and its percentage for all groups in Webpage 1. The total fixations duration for 30 Arabic (ARB) was $276.84 \mathrm{Sec}$, for 30 English (ENG) was $281.92 \mathrm{Sec}$ and for 30 Chinese $(\mathrm{CHI})$ was 289.15 $\mathrm{Sec}$. Around 5 seconds from each group was spent on saccades. The middle Ad (MidAd) received the highest fixations duration from all groups. They spent more than $20 \%$ of their fixations time on this $\mathrm{AOI}$ and it was the only $\mathrm{AOI}$ that all participants from each group noticed.

Table 1: Fixation Data for all groups in Webpage1

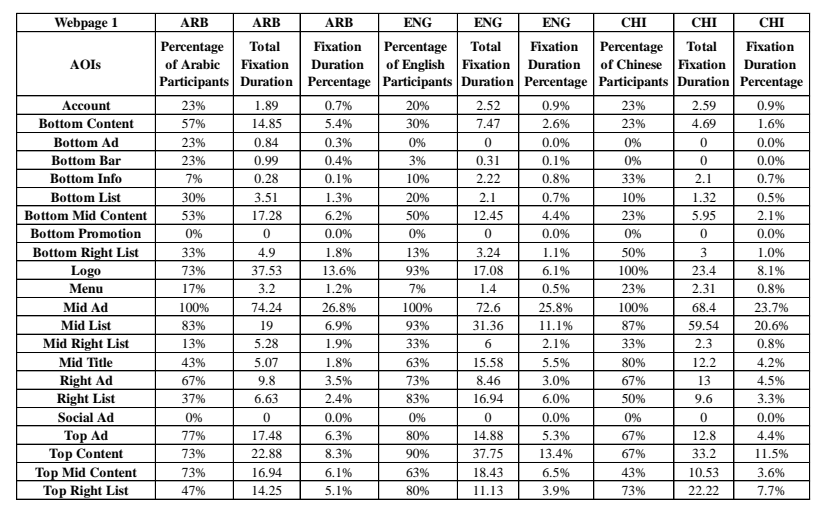

ENG spent $6.1 \%$ and $\mathrm{CHI}$ spent $8.1 \%$ of their fixation on Logo, while ARB spent double the time of fixation duration than English (13.6\%). All groups spent similar time (less than $1 \%$ of their fixations duration) on the bottom part of the webpage which includes BottomAd, BottomBar and BottomInfo AOls. $\mathrm{CHI}$ allocated $20.6 \%$ of their fixations duration on the MidList under the middle ad, while ENG and ARB tended to give less attention to this part $11.1 \%$ and $6.9 \%$ respectively. The first and second AOls that were fixated by all participants were $\operatorname{Logo}$ and MidAd AOls. Logo was the first AOI for ENG and it took them $1.5 \mathrm{sec}$ to notice it, while it was the second $\mathrm{AOI}$ for $\mathrm{ARB}$ and $\mathrm{CHI}$ and it took them (1.35 sec and $2.15 \mathrm{sec}$ respectively). What is interesting about the data is that most of the AOls located on the right hand side of the webpage such as Account and Top Right List AOls were noticed first by $\mathrm{CHI}$ followed by ENG, while ARB were the slowest group in noticing them. $23 \%$ of $A R B$ fixated on the Bottom $A d$, while no one from the English and Chinese groups notice it. Social Ad and Bottom Promo AOls located at the bottom of the page were not seen by any participants. It should be noted that $27 \%$ of ARB did not fixate on the Logo, while only $6 \%$ of ENG did not see it and all $\mathrm{CHI}$ fixated on the Logo. Figure 3 shows heatmaps that represent the aggregated fixation duration from all Arabic, English and Chinese participants in Webapge1.
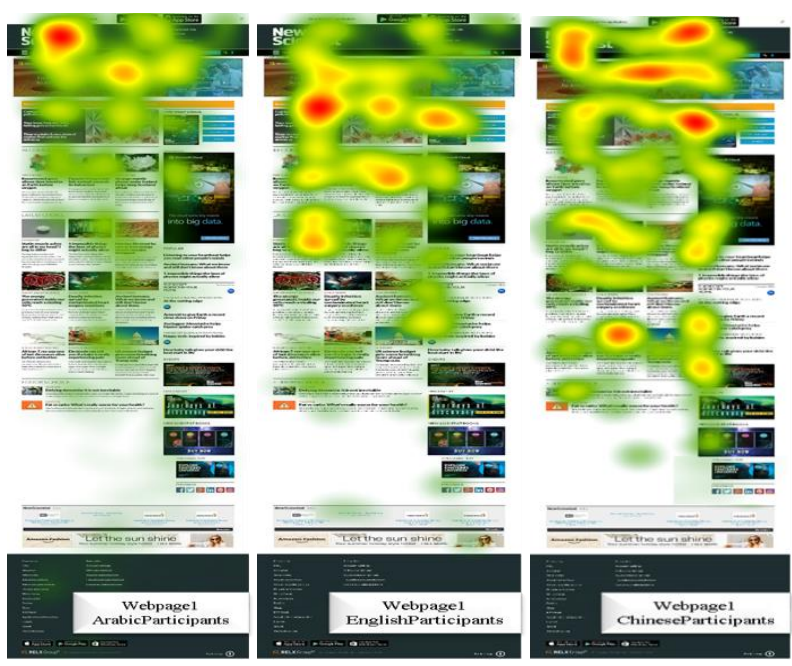

Figure 3: Heatmaps of all groups in Webpage1

Providing heatmaps helps to understand the general patterns of global users and to gain an overview of the eye movements across the different parts of the page. As can be seen from the three heatmaps above, each group had different viewing patterns. ARB tended to concentrate mostly on the top of the page and the lower their eyes went, the less attention they gave. They fixated intensively on the AOls located on the top of the page such as Logo, Menu and Mid Ad. It is obvious that ENG exhibited an F-shape pattern for reading the content of this webpage. Their fixations appeared on the right hand side more than ARB. Moreover, the middle of the page received similar fixations to the top. As well as ARB, their fixations decrease while going down the page. Regarding $\mathrm{CHI}$, their 
fixations were scattered around the page with more concentrations on the top and the middle of the page compared to ARB and ENG. The bottom part did not receive any fixation from the $\mathrm{CHI}$ users.

\subsection{Fixations Analysis and Heatmaps in Webpage 2}

The webpage was divided into 17 AOls. Table 2 presents the fixation data for all groups in Webpage2.

Table 2: Fixation Data for all groups in Webpage2

\begin{tabular}{|c|c|c|c|c|c|c|c|c|c|}
\hline Webpage 2 & & & & ENG & ENG & ENG & CHI & \begin{tabular}{|l|} 
CHI \\
\end{tabular} & $\mathrm{CHI}$ \\
\hline AOIs & $\begin{array}{l}\text { Percentage } \\
\text { of Arabi } \\
\text { Participant }\end{array}$ & \begin{tabular}{|c|} 
Total \\
Fixation \\
Duration
\end{tabular} & $\begin{array}{l}\text { Fixation } \\
\text { Duration } \\
\text { Percentage }\end{array}$ & 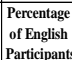 & \begin{tabular}{|c|} 
Total \\
Fixation \\
Dutarition
\end{tabular} & \begin{tabular}{|l} 
Fixation \\
Duration \\
Percentage
\end{tabular} & $\begin{array}{l}\text { Perrentage } \\
\text { of Chines } \\
\text { Partiniciana }\end{array}$ & \begin{tabular}{|c|} 
Total \\
Fixation \\
Duration
\end{tabular} & \begin{tabular}{|l|l} 
Fixation \\
Duration \\
Percentae
\end{tabular} \\
\hline Announcement & $53 \%$ & \begin{tabular}{|l|l}
14.24 \\
\end{tabular} & $5.2 \%$ & $73 \%$ & 19.36 & $7.1 \%$ & $43 \%$ & 16.38 & $5.7 \%$ \\
\hline Bottom Ad & $20 \%$ & 1.14 & $0.4 \%$ & $13 \%$ & 4.26 & $1.6 \%$ & $57 \%$ & \begin{tabular}{|l|l|}
6.97 \\
\end{tabular} & $2.4 \%$ \\
\hline Bottom Boxes & $33 \%$ & 7.8 & $2.9 \%$ & $30 \%$ & 6.75 & $2.5 \%$ & $20 \%$ & 2.64 & $0.9 \%$ \\
\hline $\begin{array}{c}\text { Bottom Content } \\
\text { Battom Ińt }\end{array}$ & $60 \%$ & $\frac{16.15}{7.92}$ & $5.9 \%$ & $80 \%$ & 17.6 & $6.4 \%$ & $43 \%$ & \begin{tabular}{|l|l|}
9.75 \\
4
\end{tabular} & $3.4 \%$ \\
\hline $\begin{array}{c}\text { Bottom Into } \\
\text { Bottom Left Ad } \\
\end{array}$ & $33 \%$ & $\frac{7.92}{4.9}$ & $\frac{2.96 \%}{1.8 \% 6}$ & $37 \%$ & $\begin{array}{l}5.84 \\
3.08 \\
\end{array}$ & $\frac{2.1 \% \%}{1.1 \%}$ & $\begin{array}{ll}33 \% \\
43 \%\end{array}$ & \begin{tabular}{|l|}
4.1 \\
4.16 \\
\end{tabular} & $\frac{1.4 \%}{1.5 \%}$ \\
\hline$\overline{B o t t o m !}$ & $80 \%$ & 24.96 & $9.1 \%$ & $80 \%$ & 27.6 & 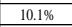 & $67 \%$ & 14 & $4.9 \%$ \\
\hline Bottom Right Ad & $23 \%$ & 1.75 & $0.6 \%$ & $20 \%$ & 2.76 & $1.0 \%$ & $43 \%$ & 3.64 & $1.3 \%$ \\
\hline$\frac{\text { Log }}{\text { Navivat }}$ & $\frac{70 \%}{67 \%}$ & 21.63 & $7.9 \%$ & $87 \%$ & 28.6 & $\frac{10.4 \%}{5 \%}$ & $67 \%$ & \begin{tabular}{|l|}
22 \\
1087
\end{tabular} & $7.7 \%$ \\
\hline $\begin{array}{l}\text { Navgation } \\
\text { Paging }\end{array}$ & $\frac{6 \% \%}{7 \%}$ & $\frac{15.8}{0.76}$ & $0.0 .3 \%$ & $\frac{61 \%}{0 \%}$ & $\frac{15.4}{0}$ & $\frac{5.6 \% \%}{0.0 \%}$ & $\begin{array}{lll}43 \% \% \\
33 \%\end{array}$ & \begin{tabular}{|l|l|}
12.87 \\
2.45 \\
\end{tabular} & $\frac{3.5 \%}{0.9 \%}$ \\
\hline 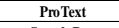 & $10 \%$ & 0.66 & & $10 \%$ & 1.98 & $0.7 \%$ & $100 \%$ & & $0.4 \%$ \\
\hline Search Box & $7 \%$ & 1.02 & $0.4 \%$ & $47 \%$ & 3.7 & $1.4 \%$ & $50 \%$ & \begin{tabular}{|l|l|}
3.01 \\
300
\end{tabular} & $1.1 \%$ \\
\hline Left 1 & $97 \%$ & $\frac{53.07}{51}$ & $\frac{19.4 \%}{187 \%}$ & $\frac{93 \%}{93 \%}$ & $\frac{50.4}{3948}$ & $18.4 \%$ & 100 & 49.8 & $17.5 \%$ \\
\hline Top Mid Content & $90 \%$ & 35.91 & $\frac{18.1 \%}{13.1 \%}$ & & 28.86 & $\frac{1.44 \%}{10.5 \%}$ & $100 \%$ & \begin{tabular}{|l|l|}
0.8 \\
15.87 \\
\end{tabular} & \begin{tabular}{|l|l|}
$21.1 \%$ \\
$5.6 \%$
\end{tabular} \\
\hline Top Right List & $67 \%$ & $\begin{array}{l}16.72 \\
\end{array}$ & $6.1 \%$ & $80 \%$ & 18.27 & $6.7 \%$ & $87 \%$ & \begin{tabular}{|l|l|l|l|} 
\\
\end{tabular} & $19.1 \%$ \\
\hline
\end{tabular}

The average fixation duration of $A R B$ and ENG participants in this webpage is the same (13 Sec), while $\mathrm{CHI}$ participants spent more fixations (14.33 $\mathrm{Sec})$. Two seconds from ARB and ENG were spent on saccades, whereas $\mathrm{CHI}$ spent only $0.5 \mathrm{Sec}$ in saccades. Top Content AOI was the most fixated area for ARB and ENG. They spent more than 18\% of their fixation duration in this part. For $\mathrm{CHI}$ participants, the most fixated AOI was Top Left Info which was above the Top Content and under the Logo and Navigation AOIs. They spent almost 22\% of their fixations duration in this part. The Logo was fixated less by ARB and $\mathrm{CHI}$ than ENG participants. Although Search Box and Bottom Right Ad AOls were located on the right hand side, ARB allocated only $1 \%$ of their fixations duration on those parts, while ENG and $\mathrm{CHI}$ participants allocated $2.4 \%$ of their fixations duration. Notably, $\mathrm{CHI}$ spent by far more time on Top Right List (19.1\%) than ARB $(6.1 \%)$ and ENG $(6.7 \%)$ participants. In addition, $\mathrm{CHI}$ participants spent around $15 \%$ of their fixation duration on AOls located at the bottom, whereas ARB and ENG participants allocated more than 23\% of their fixation duration to those AOls. However, $\mathrm{CHI}$ fixation duration on Bottom Ad was double ARB fixation duration and more than ENG fixation duration. Concerning the time to first fixation, all groups noticed first Top Left Info and then Logo AOls. However, ARB took around $1.6 \mathrm{Sec}$ to notice the first $\mathrm{AOI}$, while ENG and $\mathrm{CHI}$ took less than a second. The Bottom Right Ad was fixated first by ARB and then ENG and CHI. Pagination was seen by $33 \%$ of $\mathrm{CHI}$ and only $7 \%$ of $\mathrm{ARB}$, while no one from ENG group noticed it. Noticeably, $30 \%$ of ARB and $33 \%$ of $\mathrm{CHI}$ did not see the Logo. Moreover, only $7 \%$ of ARB saw Search Box that was located on the right hand side, while $47 \%$ and $50 \%$ of ENG and $\mathrm{CHI}$ saw this AOI. Although the Top Mid Content AOI was nearer to the top of the page than Bottom Mid Content, all participants noticed the later faster than the first. Figure 4 shows heatmaps for all groups.

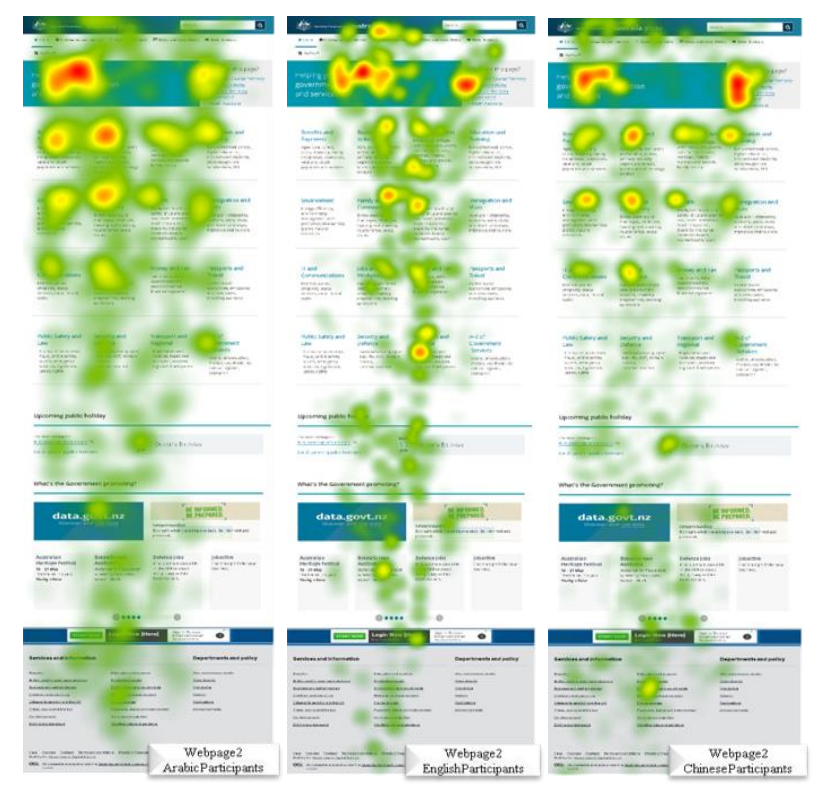

Figure 4: Heatmaps of all groups in Webpage2

The Top Left Info and Top Right List AOIs were most concentrated by ENG and $\mathrm{CHI}$. However, ARB did not pay great attention to Top Right List which was on the right hand side. ARB and $\mathrm{CHI}$ tended to read horizontally each line of the texts presented and ARB read more than $\mathrm{CHI}$ and ENG. $\mathrm{CHI}$ jumped their fixation from area to area without a connection between their fixations. ARB read with a connection between their fixations even though the area between the visual elements is a white space. ENG tended to view this webpage from the middle and moved their eyes vertically into the bottom of the page. Ignoring most of the left and right text elements.

\subsection{Fixations Analysis and Heatmaps in Webpage 1}

The webpage was divided into 19 AOIs.

Table 3: Fixation Data for all groups in Webpage3

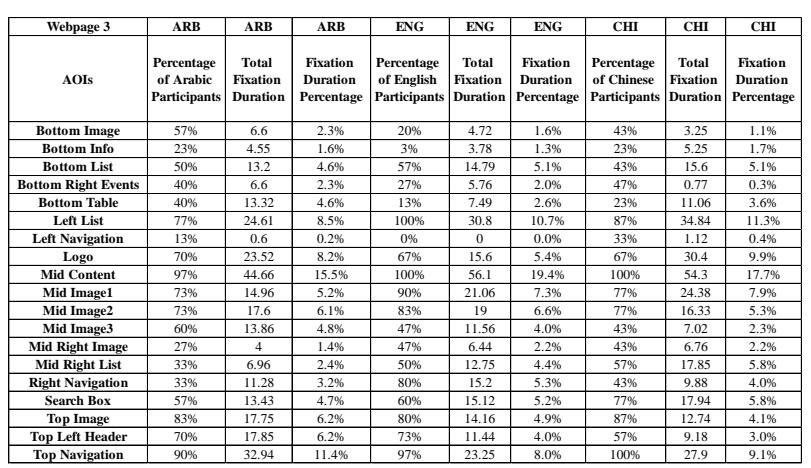


As shown in Table 3, the area that received the most fixation duration from all participants was Mid Content AOI. This AOI was a text and ENG spent $19.4 \%$ of their fixation duration on this part. $\mathrm{CHI}$ spent $17.7 \%$ and ARB spent $15.5 \%$. This webpage includes many images in different locations. Remarkably, participants from all groups spent more time on the images placed at the middle of the screen compared to those placed in the bottom and even in the top. Moreover, although there is an image on the right hand side and it was very close to those placed in the middle, all participants spent less time on that placed on the RHS. The Bottom Right Evens AOI includes three images, however, $\mathrm{CHI}$ spent only $0.3 \%$ of their fixation, ENG spent $2.2 \%$ and ARB spent a bit longer $2.3 \%$ of their fixation. Unpredictably, ARB were the lowest group spending time on the Right Navigation AOI (3.2\%), while ENG spent $(5.3 \%)$ and $\mathrm{CHI}$ spent $(4 \%)$ of their fixation. As well as Mid Right List and Mid Right Image. The first noticed AOI for ARB and ENG was Top Navigation. CHI looked first at the Search Box AOI that located on the top left corner. The second and third AOls that ARB and ENG viewed were Top Image and then Logo, while $\mathrm{CHI}$ looked to Left Navigation and then Logo as ARB and ENG. Interestingly, Top Navigation was the fifth $\mathrm{AOI}$ for $\mathrm{CHI}$, whereas it was the first $\mathrm{AOI}$ for ARB and ENG. Although the Left Navigation AOI was relatively small, $33 \%$ of $\mathrm{CHI}$ looked at it and it was the second AOI. Only 6\% of ARB saw it and no one from ENG group noticed it. What is interesting about the data is that around 30\% from each group did not see the Logo of the webpage. Moreover, even though Search Box was placed in the top left corner, $43 \%$ from ARB, $40 \%$ from ENG did not see this part but $77 \%$ of $\mathrm{CHI}$ saw it. Right Navigation AOI was the navigation bar for the webpage and it was placed on the right hand side. Surprisingly, only $33 \%$ of ARB see this area, while $80 \%$ of ENG and $70 \%$ of $\mathrm{CHI}$ fixated on this part of the page.

Generally, the areas placed on the right hand side were fixated by less ARB than ENG and $\mathrm{CHI}$. Figure 5 displays the heatmaps of all groups in Webpage3. Even though the Logo was placed on the top right corner of the screen, it was viewed intensively by all participants followed by Top Navigation and then Top Image. ARB tended to fixated mostly on the top and middle of the page with fewer fixations on the right hand side of the webpage. $\mathrm{CHI}$ fixated more on the images rather the texts and the right hand side received a great attention from $\mathrm{CHI}$ and ENG but not from ARB. Surprisingly a reverse F-shape pattern was noticed in ENG eye movements as an opposite to what they exhibited in Webpage1. Clearly ARB fixated more on the bottom part of the page than ENG and $\mathrm{CHI}$. Moreover, $\mathrm{CHI}$ tended to ignore the white spaces in the page more than ARB and ENG.

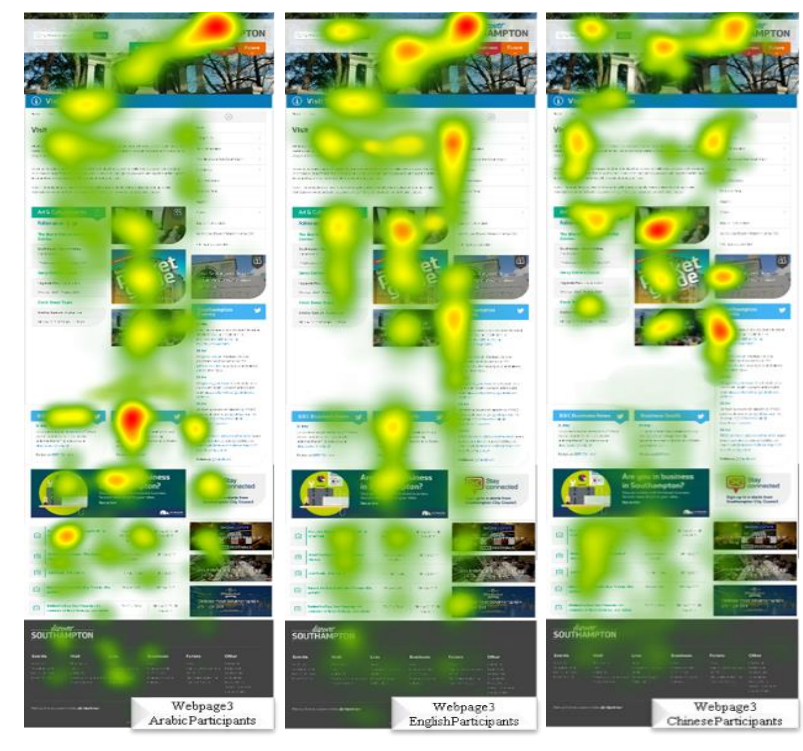

Figure 5: Heatmaps of all groups in Webpage3

\subsection{Preference Results}

This technique was adopted to elicit the general users' preferences for a webpage. This question was asked after viewing the three webpages and the answers were collected using Google Forms. The users had to choose their preferred type of webpages and justify their choices by explaining the reasons behind choosing this type over the other types of webpages. Concerning quantitative data, a chi-square test was conducted to reveal any significant association between the group type and their preferences of the webpages. There was a significant association between the preferences and group type $X^{2}(4)=23.084, p<0.001$. Interestingly, the majority of Arab group (61.5\%) preferred Webpage3 which contained images and text with mainly right hand design elements, while only $17.6 \%$ of Chinese and $22.5 \%$ of English liked this type. The image-based webpage (Webpage1) was mostly preferred by Chinese users $(72 \%)$, whereas $23.5 \%$ of Arab and $12 \%$ of English liked this webpage. The last webpage was text-based: $65.5 \%$ of English users preferred this type of webpage, while only $10.4 \%$ of Chinese and $15 \%$ of Arab users preferred this type of interface. To understand the reasons behind their choices, their comments were analysed qualitatively, and the positive and negative themes were formulated. The percentages of each theme in each webpage type are presented in the following sections.

\subsubsection{Webpage 1 Comments Analysis}

On average, the Webpage 1 that is image based received 72 positive comments and 49 negative comments from all groups. Most of the positive comments received from Chinese users. The positive comments resulted in a total of six themes and the percentage of each group is presented in Figure 6. Webpage1 was praised for its clear 
connection between images and texts $(28 \%$ of the themes), attractiveness layout (26\% of the themes), easy to navigate (15\% of the themes), consistency (14\% of the themes), clear menu (10\% of themes) and clear organised structure ( $7 \%$ of the themes).

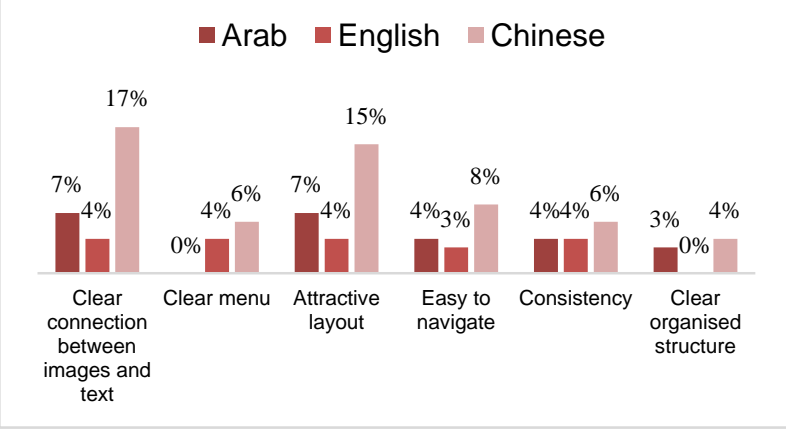

Figure 6: Positive themes in Webpage 1 across groups

The negative comments resulted in five themes as illustrated in Figure 7 and most of them were received from English users. The Webpage1 was criticised for its cluttered and busy layout ( $41 \%$ of the themes), overused of images (20\% of themes), visual overloaded (17\% of the themes), no clear guidance (16\% of the themes) and Inappropriate use of ads ( $6 \%$ of the themes).

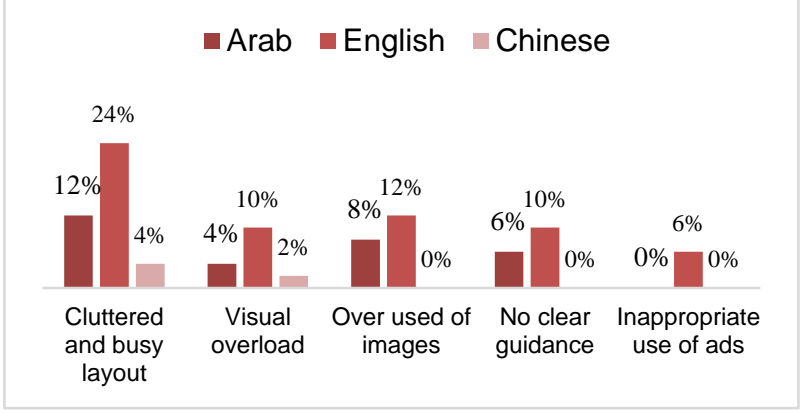

Figure 7: Negative themes in Webpage 1 across groups

\subsubsection{Webpage 2 Comments Analysis}

Webpage2, which is text-based, received 61 positive comments and 62 negative comments. As presented in Figure 8 , the positive comments resulted in a total of four themes and the majority of the positive comments were derived from English users. The users believed that the structure of the site was clear ( $44 \%$ of the themes), easy to find the information (34\% of the themes), less distracting items $(12 \%$ of themes) and the colour was balanced (10\%).

As shown in Figure 9, five themes were produced from the negative comments and received most from Chinese and Arabic users. Users said that the Webpage 2 not attractive for foreign users $(37 \%$ of the themes), had too heavy text $(21 \%$ of the themes), dull interface (19\% of the themes), lack of images (18\% of the themes) and subject feelings ( $5 \%$ of the themes).

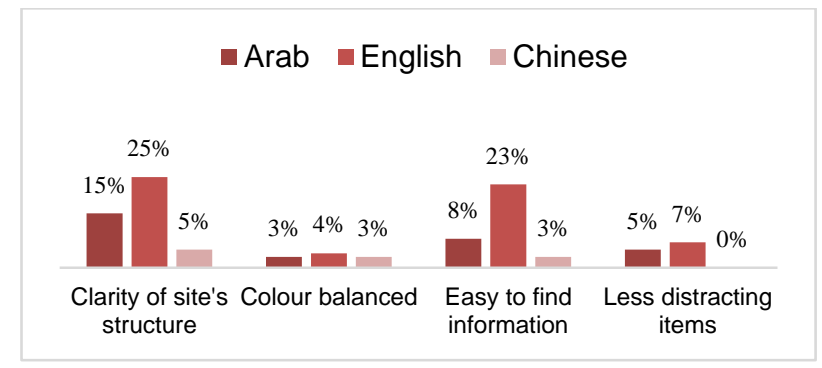

Figure 8: Positive themes in Webpage 2 across groups

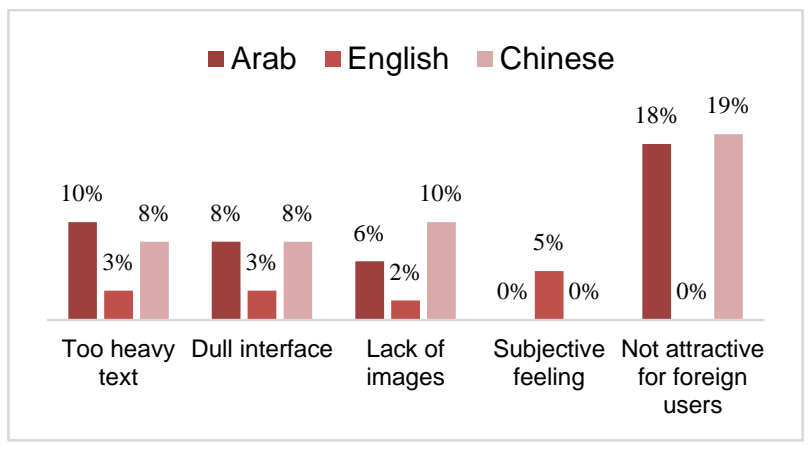

Figure 9: Negative themes in Webpage 2 across groups

\subsubsection{Webpage 3 Comments Analysis}

The Webpage3 has a mixture of images and text. It has unusual default design of English webpages. This webpage received 58 positive comments and 48 negative comments. The positive comments resulted in four themes and Arab users delivered the most positive comments. As presented in Figure 10, participants felt that the layout was organised and balanced (59\% of the themes), the toolbar was useful (15\% of the themes), the navigation's position is more acceptable $(14 \%$ of the themes) and easy to navigate through the page $(12 \%$ of the themes).

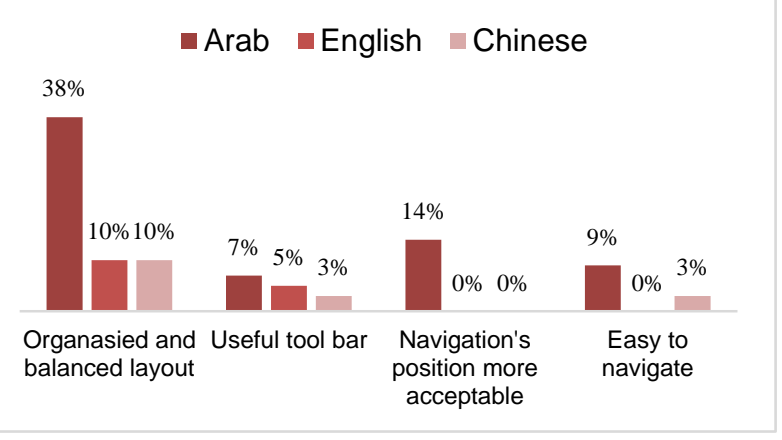

Figure 10: Positive themes in Webpage 3 across groups

Five themes were formulated based on the 48 negative comments which were mostly received from English users. As presented in Figure 11, participants believed that the design of this webpage was messy (27\% of the themes), the location of the navigation bar was inappropriate (23\% of themes), inconsistency $(15 \%$ of the themes) and the design of this webpage was distracting the attention ( $14 \%$ of the themes). 


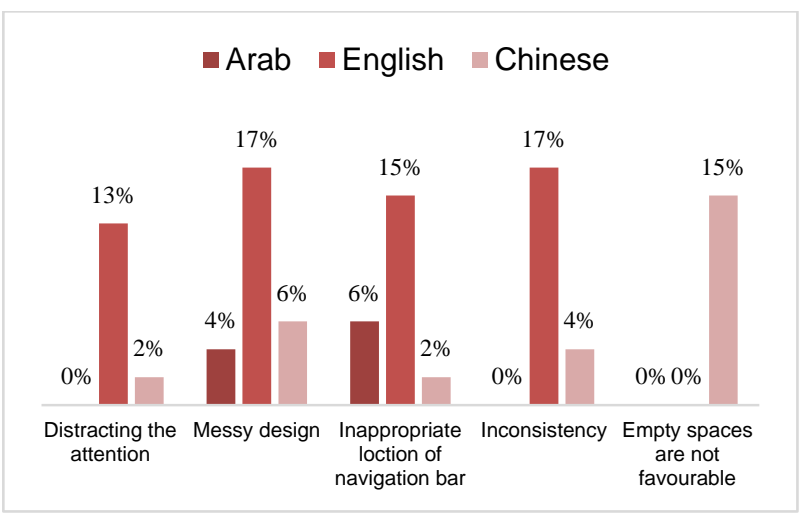

Figure 11: Negative themes in Webpage3 across groups

\section{DISCUSSION AND CONCLUSIONS}

The study uncovered strong differences between the three groups while viewing different types of webpage. The logo of the three webpages whether placed on the right or on the left was noticed from all groups as the first, second or third AOI. This was as predicted based on (Arroyo et al. 2006), who claimed that the logo is one of three main parts of the webpage that users usually interact most. An important contribution in this study is that elements located on the right hand side such as right navigation in Webpage 3 and search box in Webpage2, are noticeable by English and Chinese users. In contrast, Arab users were the slowest group to pay attention to most of those items and the lowest group fixated on them. This was a surprise, as it suggests that the language reading direction (R-L) has no influence on their eye movements. The likelihood of seeing ads at the top of the page is more than the ads placed at the bottom of the page. Generally, all participants had a bias toward the elements placed in the top of the page rather the bottom ones. Consumers' perception of brand strength and relevancy of sources is influenced by items positions and in sequenced order that trigger knowledge implications (Dou et al. 2010).

Furthermore, the chance to notice the ads in the text-based webpage is more than webpage with many images. For example, the bottom ad in Webpage 1 was seen by only $23 \%$ of Arab users, while the bottom ad in Webpage2 was seen by all participants. In addition, the middle ad in Webpage 1 was seen by all participants and it was the most fixated area in this webpage. This may be because this ad contained human faces and, instinctively, human faces hold a lot more appeal to people over objects (Tullis et al. 2009).

The viewing patterns and fixations distributions on the three webpages were different for the three groups due to the differences in their cognitive style. This was shown by previous studies. In an empirical study carried out by (Masuda \& Nisbett
2001; Dong \& Lee 2008), Chinese users were found to have short periods of fixation on various areas of a website navigating more frequently through various sections of the whole page while their western counterparts concentrated their fixations more on specific areas. In another study by (Qutub 2008) Arab users were found to be relatively more holistic minded like Chinese, rather than analytic minded like Westerners.

Arab users usually concentrated on the top with a considerable attention to the bottom. However, unpredictably items placed on the right hand side are usually neglected. Chinese users scattered their fixation around the page especially in Webpage 1 with less attention towards the bottom. They appeared to read intensively from the top upper left to the top upper right and then downing their eyes in a similar manner. Therefore, we noticed that Chinese see more elements that placed on the upper left and upper right than Arab and English users. In terms of English users, our findings is in line with (Nielsen 2006) about the Fshape while reading the web content. The F-shape was observed in Webpage1, while interestingly English users applied a reverse $\mathrm{F}$-shape in Webpage 3 as most of the elements were placed on RHS. In Webpage2, English mostly read the textbased webpage vertically from the middle in contrast to Arab and Chinese users where they read from the left to the right for each line especially for Arab. (Marcos et al. 2013) noted that Arab tended to read more results in a completed way more than Westerners did.

Concerning the preferences differences, although Arab users paid less attention to the right hand side elements in Webpage3, the majority of them liked this type when it was shown to them with other tested webpages after the eye tracking session to choose from. This means they would be satisfied when English website is designed according to their reading direction even though our findings approved that their scanning patterns are not affected with their reading direction. The majority of Chinese participants preferred the image-based webpage, while the majority of English participants preferred the text-based webpage. This finding is consistent with studies by (Fraternali \& Tisi 2008; Dong \& Lee 2008) which found that Chinese users preferred high information density on webpages with wealth of non-textual information such as images, while Western users favoured clarity with minimum information available.

This study has raised a main issue for a topic that is principally unexplored and it is of importance to research conducted in human-computer interaction $(\mathrm{HCl})$, web design as well as user experience (UX). In future work, we propose to extend the media to include videos, animations, and also to extend the range of user tasks. 


\section{REFERENCES}

Ahmed, T., Mouratidis, H. \& Preston, D., 2009. Website Design Guidelines: High Power Distance and High-Context Culture. International Journal of Cyber Society and Education, 2(1), pp.47-60.

Alex, W., 2014. Cataloging the World: Paul Otlet and the Birth of the Information Age, Oxford University Press.

Alsaffar, M., 2018. Online Visual Behaviour and Satisfaction: a Comparative Study of English, Arabic and Chinese Users. University of Brighton, UK.

Arroyo, E., Selker, T. \& Wei, W., 2006. Usability Tool for Analysis of Web Designs Using Mouse Tracks. In CHI'06 Extended Abstracts on Human Factors in Computing Systems. pp. 484-489.

Badre, A., 2001. The Effects of Cross Cultural Interface Design Orientation on World Wide Web User Performance. Georgia Institute of Technology, pp.1-30.

Bojko, A., 2013. Eye Tracking the User Experience: A Practical Guide to Research M. Justak, ed., Brooklyn, New York: Louis Rosenfeld.

Bonnardel, N., Piolat, A. \& Le Bigot, L., 2011. The impact of colour on Website appeal and users' cognitive processes. Displays, 32(2), pp.69-80.

Brady, L. \& Phillips, C., 2003. Aesthetics and Usability: A Look at Color and Balance. Software Usability Research Laboratory. Available at: http://usabilitynews.org/ aesthetics-and-usability-a-look-at-color-andbalance/ [Accessed December 5, 2017].

Brandtzæg, P.B., Heim, J. \& Karahasanović, A., 2011. Understanding the new digital divide A typology of Internet users in Europe. International Journal of Human-Computer Studies, 69(3), pp.123-138.

Cairns, P. \& Cox, A., 2008. Research methods for human-computer interaction, Cambridge: Cambridge University Press.

Cowen, L., Ball, L.J.. \& Delin, J., 2002. An Eye Movement Analysis of Web Page Usability. In People and Computers XVI-Memorable Yet Invisible. London: Springer London, pp. 317335.

Cyr, D., 2008. Modeling Website Design across Cultures : Relationships to Trust, Satisfaction and E-loyalty ( Forthcoming Journal of Management Information Systems ) Modeling Website Design across Cultures :

Relationships to Trust, Satisfaction and Eloyalty. Journal of Management Information Systems, 24, pp.47-72.

Cyr, D., 2013. Website design, trust and culture: An eight country investigation. Electronic Commerce Research and Applications, 12(6), pp.373-385.
Cyr, D., Head, M. \& Larios, H., 2010. Colour appeal in website design within and across cultures: A multi-method evaluation. International Journal of Human Computer Studies, 68(1-2), pp.1-21.

Deng, L. \& Poole, M.S., 2010. Affect in Web Interfaces: A Study of the Impacts of Web Page Visual Complexity and Order. MIS Quarterly, 34(4), p.711.

Dong, Y. \& Lee, K.P., 2008. A cross-cultural comparative study of users' perceptions of a webpage: With a focus on the cognitive styles of Chinese, Koreans and Americans. International Journal of Design, 2(2), pp.19-30.

Dou, W. et al., 2010. Brand Positioning Strategy Using Search Engine Marketing. MIS Quarterly, 34(2), p.261.

Faiola, A. \& Matei, S. a, 2005. Cultural Cognitive Style and Web Design: Beyond a Behavioral Inquiry into Computer-Mediated Communication. Journal of ComputerMediated Communication, 11(1), pp.375-394.

Fraternali, P. \& Tisi, M., 2008. Identifying cultural markers for web application design targeted to a multi-cultural audience. Proceedings - 8th International Conference on Web Engineering, ICWE 2008, pp.231-239.

Geissler, G.L., Zinkhan, G.M. \& Watson, R.T., 2006. The Influence of Home Page Complexity on Consumer Attention, Attitudes, and Purchase Intent. Journal of Advertising, 35, pp.69-80.

Hartmann, J., Sutcliffe, A. \& De Angeli, A., 2007. Investigating attractiveness in web user interfaces. Proceedings of the SIGCHI conference on Human factors in computing systems - CHI '07, p.387.

Head, M. \& Cyr, D., 2013. Website design in an international context: The role of gender in masculine versus feminine oriented countries. Computers in Human Behavior, 29(4), pp.1358-1367.

Hernandez, M.D. et al., 2017. Escaping the corner of death? An eye-tracking study of reading direction influence on attention and memory. Journal of Consumer Marketing, 34(1), pp.1-10.

Hoyer, W.D. \& MacInnis, D.J., 1997. Consumer Behavior, Houghton Mifflin.

Hsieh, H.C., 2008. A New Model for Cross-cultural Web Design. Brunel University.

Jacko, J., 2012. The human-computer interaction handbook : fundamentals, evolving technologies, and emerging applications Third., CRC Press.

Jacob, R.J.K. \& Karn, K.S., 2003. Eye tracking in Human-Computer interaction and usability research: Ready to deliver the promises. In The Mind's Eye: Cognitive and Applied Aspects of Eye Movement Research. Oxford: Elsevier Science BV., pp. 573-605. 
Josephson, S. \& Holmes, M.E., 2002. Attention to repeated images on the World-Wide Web: another look at scanpath theory. Behavior Research Methods, Instruments, \& Computers, 34(4), pp.539-548.

Lazar, J., Feng, J.H. \& Hochheiser, H., 2017. Research Methods in Human-Computer Interaction Second Edi., Morgan Kaufmann.

Lee, Y. \& Kozar, K.A., 2012. Understanding of website usability: Specifying and measuring constructs and their relationships. Decision Support Systems journal, 52(2).

Li, H., Sun, X. \& Zhang, K., 2007. Culture-Centered Design Cultural Factors in Interface Usability and Usability Tests. In International Conference on Software Engineering, Artificial Intelligence, Networking, and Parallel/Distributed Computing. pp. 843-847.

Lindgaard, G. et al., 2011. An exploration of relations between visual appeal, trustworthiness and perceived usability of homepages. ACM Transactions on ComputerHuman Interaction, 18(1), pp.1-30.

Marcos, M. et al., 2013. Cultural Differences on Seeking Information: An Eye Tracking Study. In CHl' 13. Paris, France: ACM.

Marcos, M. \& Gavin, F., 2015. Effect of Snippets on User Experience in Web Search. In Proceedings of the XVI International Conference on Human Computer Interaction. Vilanova i la Geltrú, Spain: ACM, p. 47.

Masuda, T. \& Nisbett, R.E., 2001. Attending holistically versus analytically: comparing the context sensitivity of Japanese and Americans. Journal of personality and social psychology, 81(5), pp.922-34.

Nielsen, J., 2006. F-Shaped Pattern For Reading Web Content. Nielsen Norman Group. Available at: https://www.nngroup.com/articles/f-shapedpattern-reading-web-content/.

Palmer, J.W. \& W., J., 2002. Web Site Usability, Design, and Performance Metrics. Information Systems Research, 13(2), pp.151-167.

Palmer, S.E. \& Schloss, K.B., 2010. Human preference for individual colors. Proceedings of Society of Photo-Optical Instrumentation Engineers (SPIE) Conference, 107(19), p.752718.

Pan, B. et al., 2004. The determinants of web page viewing behavior: an eye-tracking study. Proceedings of the ETRA '04 Symposium on Eye Tracking Research and Applications, 1(212), pp.147-154.

Purchase, H.C., 2012. Experimental HumanComputer Interaction: A Practical Guide with Visual Examples, New York, USA: Cambridge University Press.

Qutub, J.A., 2008. A Cross-Cultural Comparison of Cognitive Styles in Arab and American Adult
Learners Using Eye-Tracking to Measure Subtle Differences. George Mason University.

Rayner, K. et al., 2006. The effect of word frequency, word predictability, and font difficulty on the eye movements of young and older readers. Psychology and Aging, 21(3), pp.448-465.

Reinecke, K. et al., 2013. Predicting Users' First Impressions of Website Aesthetics With a Quantification of Perceived Visual Complexity and Colorfulness. In $\mathrm{CHI}$ Changing Perspectives.

Reinecke, K., Arbor, A. \& Gajos, K.Z., 2014. Quantifying Visual Preferences Around the World. In Proceedings of the SIGCHI Conference on Human Factors in Computing Systems. Toronto, ON, Canada: ACM, pp. $11-20$.

Reinecke, K. \& Bernstein, A., 2013. Knowing What a User Likes: A Design Science Approach to Interfaces that Adapt to Culture. MIS Quarterly, 37(2), pp.427-453.

Resort, C. et al., 2009. Culture 's Impact on Arabic Website Design. In Proceedings of HumanComputer Interface International. pp. 1-9.

Schenkman, B.N. \& Jonsson, F.U., 2000. Aesthetics and preferences of webpages. Behaviour \& Information Technology, 19(5), pp.367-377.

Seckler, M., Opwis, K. \& Tuch, A.N., 2015. Linking objective design factors with subjective aesthetics: An experimental study on how structure and color of websites affect the facets of users' visual aesthetic perception. Computers in Human Behavior, 49, pp.375389.

Sharma, C. \& Dubey, S.K., 2014. Analysis of Eye Tracking Techniques in Usability and $\mathrm{HCl}$ Perspective. In International Conference on Computing for Sustainable Global Development (INDIACom). pp. 607-612.

Tractinsky, N., Katz, A.. \& Ikar, D., 2000. What is beautiful is usable. Interacting with Computers, 13(2), pp.127-145.

Tuch, A.N. et al., 2012. Is beautiful really usable? Toward understanding the relation between usability, aesthetics, and affect in $\mathrm{HCl}$. Computers in Human Behavior, 28(5), pp.1596-1607.

Tuch, A.N. et al., 2009. Visual complexity of websites: Effects on users' experience, physiology, performance, and memory. International Journal of Human-Computer Studies, 67(9), pp.703-715.

Tullis, T., Siegel, M. \& Sun, E., 2009. Are people drawn to faces on webpages? In Conference on Human Factors in Computing Systems. p. 5.

Tzanidou, E., 2006. Evaluating usability of ecommerce sites by tracking eye movements. 
Vatrapu, R. \& Pérez-Quiñones, M.A., 2006. Culture and Usability Evaluation: The Effects of Culture in Structured Interviews. Journal of Usability Studies, 1(4), pp.156-170.

Venkatesh, V. et al., 2003. User Acceptance of Information Technology: Toward a Unified View. MIS Quarterly, 27(3), pp.425-478.

Wang, Q. et al., 2014. An eye-tracking study of website complexity from cognitive load perspective. Decision Support Systems, 62, pp.1-10.

Web Technology Surveys, 2018. Usage Statistics of Content Languages for Websites. Available at:

http://w3techs.com/technologies/overview/con tent_language/all [Accessed March 18, 2018]. 